HAJER TABAKH

\title{
LA SOUFFRANCE AMOUREUSE CHEZ MARIE NDIAYE
}

L'amour est sans doute un thème fondamental dans l'œuvre ndiayienne, non pas qu'il soit vécu comme une idylle entre deux cœurs destinés à écrire une histoire heureuse, mais un amour inévitablement voué à l'échec et à la perdition de soi. Malgré l'apparence hétéroclite du matériau narratif, le lecteur a cette troublante impression de relire à chaque fois cette même histoire d'amour enclin fatalement au naufrage.

Tant de femmes et hommes malheureux peuplent l'univers de Marie NDiaye. Le bonheur amoureux semble être inexprimable, ou du moins même s'il existe est très rapidement périssable; mais la souffrance, elle, lorsqu'elle est vécue intensément, s'empare de tout le corps du personnage et c'est l'écriture qui prend en charge toute la douleur ressentie. Sachant que chez Marie NDiaye, les personnages vivent par l'intermédiaire de leurs corps, surface de captation du monde environnant et abîme émotionnel, il s'agira donc dans le cadre de cet article de déceler les expressions affectives et d'étudier les mécanismes du langage émotionnel. Nous verrons également comment l'affect façonne le parcours du personnage ndiayien et comment il modèle son environnement narratif.

La rupture amoureuse comme déclencheur de la souffrance et les sensations psychologiques et physiques qui lui sont liées

Un corps qui se sépare d'un autre, qui établit une distance et s'éloigne, tel est donc le leitmotiv qui revient dans chaque texte de Marie NDiaye. Cet éloignement engendre chez le personnage délaissé et qui aime encore, une souffrance qui prend parfois des formes extrêmes. C'est la "souffrance viscérale »" la souffrance morale, qui retiendra toute notre attention car elle s'accompagne de modifications musculaires et elle peint à grands traits la transformation du corps suite à l'échec amoureux. Puisque le corps physique est indissociable de sa réalité affective, une dialectique sera ainsi instaurée entre le corps souffrant et la psyché du personnage sur la base d'une analyse thématique. Nous emprunterons en ce sens certains outils d'analyse à la narratologie et à la psychanalyse.

Selon une perspective psychanalytique, notamment d'après Julia Kristeva, la souffrance est à déchiffrer dans les bouleversements du corps, dans les symptômes et démonstrations extérieures ${ }^{2}$. La perspective de Juan-David Nasio est plus

\footnotetext{
${ }^{1}$ Daniel Schurmans, L'homme qui souffre, Paris, PUF, 2010, p. 28.

2 «L'appel, son appel, me déborde d'un flux où se mêlent des bouleversements du corps (ce qu'on appelle des émotions) et une pensée en tourbillon, aussi vague, souple, prête à percer ou à épouser celle de l'autre, que vigilante, éveillée lucide dans son élan... vers quoi? Vers un destin,
} 
éclairante à ce sujet car elle inclut la tendance à l'action du corps sous l'influence de l'émotion. Elle précise que la douleur psychique « commence avec une rupture, se poursuit avec la commotion psychique que la rupture déclenche, et culmine avec une réaction défensive du moi pour parer à la commotion $»^{3}$. Dans les récits ndiayiens, ces trois étapes correspondent à trois niveaux: la déclaration de la rupture par l'un des conjoints, les sensations psychologiques et physiques liées à l'annonce de la rupture et la réaction du corps.

Dans le roman Ladivine, c'est le couple Clarisse - Richard Rivière qui se sépare après vingt-cinq ans de mariage. Quand Richard quitte le domicile conjugal, c'est une véritable descente aux enfers qui attend Clarisse. L'annonce de la séparation a d'ailleurs fait l'effet d'une bombe :

...je vais m'en aller, je n'ai plus envie de vivre ici, avec toi, ce qui ne signifie pas que je ne t'aime plus, tu seras toujours ma... Une sirène se mit à hurler mais les lèvres de Richard Rivière continuaient de bouger et sa main de presser doucement celle de Clarisse, et cette absence de réaction la surprit avant qu'elle réalisât que ce bruit épouvantable provenait de son propre cerveau ${ }^{4}$.

La menace de la séparation est matérialisée par un puissant signal sonore intérieur. Le moi est plongé dans une détresse extrême et ébranle tous les centres nerveux, ce qui provoque le déclenchement d'une alarme intérieure. Métaphore d'un cri et non d'un appel au secours car inaudible pour Richard, l'être aimé, mais assourdissant et insoutenable pour l'émettrice, ce signal sonore ancre dans la chair ce que Juan-David Nasio appelle une "douleur hallucinée »". La douleur s'enracine dans la chair de la protagoniste et s'y inscrit entièrement de telle sorte que « le souvenir de ce qu'il en était lui revenait de plein fouet quand elle s'était abandonnée à rêvasser de cette façon, ou encore au petit matin, et elle s'éveillait la figure trempée de pleurs ${ }^{6}$. Cette rupture est tellement brutale et violente qu'elle " suscite immédiatement une souffrance intérieure vécue comme un arrachement de l'âme, comme un cri muet jaillissant des entrailles ${ }^{7}$. Le système psychique est déstructuré et toutes les fonctions du corps se détraquent: nausées, agitations, perte d'équilibre, pleurs, sont les symptômes du sombre chagrin qui investissent tout l'être :

implacable et aveugle comme une programmation biologique, comme la voie de l'espèce...Corps soufflé, présent dans tous ses membres par une absence délicieuse - voix tremblante, gorge sèche, œil flou de lueur, peau rosée ou moite, cœur palpitant..." (Julia Kristeva, Histoires d'amour, Paris, Denoël, 1983, p. 13).

${ }^{3}$ Juan-David Nasio, La douleur d'aimer, Paris, Payot, 2005, p. 28.

${ }^{4}$ Marie NDiaye, Ladivine, Paris, Gallimard, 2013, p. 93.

${ }^{5}$ Juan-David Nasio, Le livre de LA DOULEUR et de L'AMOUR, Paris, Payot \& Rivages, 1996, p. 203.

${ }^{6}$ Marie NDiaye, Ladivine, p. 95.

${ }^{7}$ Juan-David Nasio, La douleur d'aimer, p. 95. 
Dans le même temps une affreuse sensation de nausée la fit gémir malgré elle. Croyant sans doute qu'elle allait tomber, Richard Rivière la prit dans ses bras. (...) Elle se sentait si mal cependant, si terriblement mal que sa gêne refluait, repoussée par la douleur pleine d'écœurement, de mépris et d'horreur de tout qui avait gagné son corps entier, qui faisait trembler ses membres et tentait vainement d'écarter sa poitrine pour sortir, mais sa chair dense et ferme s'était refermée sur la douleur comme les murs de la maison sur les paroles irrévocables et rien ne serait plus capable, songeait-elle, de l'en extraire ${ }^{8}$.

Ce passage pose les relations entre la souffrance et son langage et débouche immanquablement sur la question du sens. Le corps exprime sa souffrance psychique et il existe une augmentation excessive dans la souffrance, du supportable à l'insupportable, traduite par les figures de gradation et d'intensité.

Dépositaire de l'annonce désastreuse, le corps ne préserve plus son homéostasie et absorbe en son for intérieur les mots annonçant une rupture irrémédiable. Le moi, siège de la perception-conscience, est troublé par l'évocation de cette phrase: "Je vais m'en aller, je quitte la maison »" Si initialement Clarisse semble ne pas réagir, d'où la réitération de l'annonce de la rupture par Richard Rivière (« Je ne suis pas sûr que tu m'aies compris (...), je vais m'en aller, je n'ai plus envie de vivre ici, avec toi... ${ }^{10}$ ), elle saisit ensuite la portée de la phrase formulée et intercepte la valeur de ces mots par l'intermédiaire de son corps, métaphore de la pensée : «...les mots et leur signification brutale avaient pénétré sa peau sans défense $»^{11}$. Tel un coup de poignard ou « un coup de foudre ${ }^{12}$ pour reprendre des termes freudiens, perçant la surface cutanée, les mots pénètrent en profondeur, perturbent et dérèglent la stabilité du milieu intérieur et y provoquent des troubles.

L'extérieur corporel ne se présente pas uniquement comme une surface poreuse, perméable aux agressions et menaces environnantes mais également comme une matière-éponge; autrement dit le corps a la propriété d'absorber « la quantité d'énergie externe $»^{13}$ et de les emprisonner en son sein. La peau, lieu des perceptions externes et internes, s'empare de ces mots mortels qui commencent 《calmement, méticuleusement, leur ravage ${ }^{14}$.

C'est donc à travers le corps que se manifeste la douleur amoureuse de Clarisse. La rupture tout comme la rencontre de l'ex-conjoint sont vécues comme

\footnotetext{
${ }^{8}$ Marie NDiaye, Ladivine, p.93.

${ }^{9}$ Ibidem, p. 91.

${ }^{10}$ Ibidem, pp. 92-93.

${ }^{11}$ Ibidem, p. 91

${ }^{12}$ Selon Freud, « la quantité d'énergie externe produit un frayage et il est certain que la douleur laisse derrière elle des frayages permanents dans les neurones du souvenir, à la manière d'un coup de foudre », «Esquisse d'une psychologie scientifique» (Sigmund Freud, La naissance de la psychanalyse, Paris, PUF, 1991, p. 326).

${ }^{13}$ Juan-David Nasio, La douleur d'aimer, p. 95

${ }^{14}$ Marie NDiaye, Ladivine, p. 91.
} 
des expériences extrêmement éprouvantes pour la majorité des personnages ndiayiens. Dans Papa doit manger, le personnage de Maman, qui rencontre pour la première fois son ex-mari Ahmed parti depuis dix ans, extériorise les signes d'une perturbation incoercible :

Voilà que, tout à l'heure, je reconnais depuis le hall la voix de mon mari parti pendant dix ans, j'ai peur, je tremble, je m'assieds dans un coin de l'escalier en me demandant si je serais capable de monter. Et je me lève tout de même et mes genoux cognent l'un contre l'autre et j'ai envie de vomir et je me dis, tout en grimpant marche après marche très lentement, je me dis : Quelle femme convenable tu fais... ${ }^{15}$.

Le choc des retrouvailles est intensément vécu et se déploie dans tout un champ lexical de la paralysie : "Mais je l'aime et j'en suis glacée, anéantie... ${ }^{16}$. La rencontre lui cause une émotion si forte que le mouvement du corps en est suspendu, d'où l'idée d'une relation de causalité entre l'affect et la gestuelle corporelle explicitement décrite par Freud : «Un événement tel qu’un traumatisme extérieur produira toujours une grave perturbation dans l'économie énergétique de l'organisme et mettra en mouvement tous les moyens de défense ${ }^{17}$.

Après la phase de la commotion, s'en suit l'étape de la réaction défensive du moi. Comme pour pallier cette perte irrémédiable et comme pour inhiber la douleur liée au traumatisme de la séparation, les personnages ayant perdu l'être aimé font revivre dans leur inconscient l'image de leur objet d'amour. L'absence de l'autre dure et c'est celui qui reste qui tient le discours amoureux et qui "manipule $»^{18}$ l'absence de l'aimé. Dès lors apparaissent constamment dans le texte ndiayïen des anamnèses qui renseignent le lecteur sur l'état d'avancement psychologique du personnage. Pour la veuve sans enfants Khady Demba, ces images font émerger, sur un mode d'une perception diffuse et rêveuse, " le visage flou de son mari qui lui souriait d'un éternel et charitable sourire ${ }^{19}$. Ayant perdu simultanément son époux de façon prématurée et sa situation économique, le personnage féminin ne cesse de se remémorer à chaque étape de son parcours et à chaque difficulté « la bonne et nébuleuse figure de son mari $»^{20}$. Ces irruptions imaginées l'accompagnent comme un miroir apaisant et protecteur et affleurent dans des moments de doute ou de choix, l'interrogeant sur son bien-être et lui rappelant sa vie confortable passée aux côtés de son mari.

\footnotetext{
${ }^{15}$ Marie NDiaye, Papa doit manger, Paris, Minuit, 2003, p. 29.

${ }^{16}$ Ibidem, p. 35.

17 Sigmund Freud, «Au-delà du principe de plaisir », in Essais de psychanalyse, Paris, Payot, 1971, p. 37.

18 «L'absence dure, il me faut la supporter. Je vais donc la manipuler : transformer la distorsion du temps en va-et-vient, produire du rythme, ouvrir la scène au langage [...]» (Roland Barthes, "Fragments d'un discours amoureux », in Euvres complètes, V, Paris, Seuil, 1977, p. 43).

${ }^{19}$ Marie NDiaye, Trois femmes puissantes, Paris, Gallimard, 2009, p. 253.

${ }^{20}$ Ibidem, p. 258.
} 
Quoi qu'il en soit, l'apparition de l'image de l'objet d'amour perdu est manifestement une émanation de la douleur prégnante du personnage : le moi se hisse et s'attache à se représenter l'autre qu'il a auparavant choisi, et s'y accroche désespérément pour tenter de ne pas sombrer dans la mélancolie.

L'image de l'objet élu par choix narcissique, souvent magnifiée, semble foncièrement investie d'affects puisqu'elle dresse généralement un portrait laudatif de l'être aimé.

Dans Ladivine, Clarisse multiplie les images du passé même après des années de leur rupture, se remémore du " plaisir sensuel qu'elle avait toujours à caresser le beau visage sain de Richard Rivière $»^{21}$, se rappelle de « la paix de sa vie d'avant, quand Richard Rivière vivait encore auprès d'elle $»^{22}$, de son « irréductible amour pour Richard Rivière $»^{23}$. La perte est cruellement ressentie par Clarisse qui «pleurait sans s'en rendre compte ${ }^{24}$ car tout lui manque : l'intimité partagée (« le corps de Richard Rivière lui était aussi familier que le sien propre $\aleph^{25}$ ), l'odeur de son mari ( «l'odeur fraîche, enfantine qu'elle connaissait si bien ${ }^{26}$ ), sa tendresse («Richard Rivière, attentionné et délicat comme il l'était toujours $»^{27}$ ).

Mais quel est le destin de cette souffrance amoureuse?

Le destin de la souffrance amoureuse ou comment l'affect façonne le parcours du personnage ndiayien

Brisés, les personnages empruntent une trajectoire descendante menant vers déréliction. La trame narrative semble se présenter comme un compte à rebours dramatique qui mène le personnage à sa perte. Suite à cette douloureuse rupture, Clarisse «se pensait frappée mortellement et n'avait plus qu'à attendre l'échéance... $»^{28}$. Face au bouleversement qu'a causé la perte de l'objet aimé, le soin accordé à l'image de soi se dégrade : «Elle avait un souci moins grand de son aspect, de ses vêtements et le linge qu'elle portait n'était plus aussi rigoureusement propre qu'auparavant. Ses pieds étaient secs et jaunâtres dans ses sandales ${ }^{29}{ }^{2}$. La perte de l'amour est similaire à la perte de l'intégrité corporelle, ou encore de l'intégrité de l'image du corps. En effet, la douleur de Clarisse vient du fait qu'une blessure narcissique s'est réactivée. Le narcissisme de la protagoniste est entaché par la pensée qu'elle n'est pas digne d'être aimée. Si initialement le choix de

\footnotetext{
${ }^{21}$ Marie NDiaye, Ladivine, p. 113.

${ }^{22}$ Ibidem, p. 114.

${ }^{23}$ Ibidem, p. 121.

${ }^{24}$ Ibidem, p. 97.

${ }^{25}$ Ibidem, p. 95.

${ }^{26}$ Ibidem, p. 93.

${ }^{27}$ Ibidem, p. 96.

${ }^{28}$ Ibidem, p. 98.

${ }^{29}$ Ibidem, p. 99.
} 
l'objet amoureux s'est porté sur Richard Rivière, c'est parce qu'il représente sa propre personne, ou du moins ce qu'elle imagine être :

Il lui semblait reconnaître l'odeur de sa peau, la forme de ses ongles, la manière dont se tendaient, sous le tissu du pantalon, les muscles de ses jambes quand il freinait ou accélérait, et qu'elle aimait tout cela, qu'elle aimait chaque fragment de sa réalité charnelle comme elle reconnaissait et aimait son propre corps ${ }^{30}$.

Il représente son reflet et elle se passionne pour sa propre image, ce qui la conduira inévitablement à la mort, résultat d'une faute narcissique. Ce que la protagoniste a perdu avec le départ de Richard, c'est tout d'abord l'image d'ellemême qu'il lui permettait de chérir. «Ce qu'(elle) a perdu avant tout, c'est l'amour d'(elle-même) que l'autre rendait possible. C'est-à-dire que ce qui est perdu, c'est le moi idéal, ou plus exactement mon moi idéal lié à la personne qui vient de disparaitre $»^{31}$.

C'était par et à travers l'amour que Clarisse se sentait vivre au monde. Se sachant aimée lui renvoyait une image positive d'elle mais désormais privée de cet amour qui lui rehaussait son image à travers le regard de l'autre, Clarisse se rend coupable d'une faute et endosse la responsabilité dans cette rupture. Honte, sousestime de soi et culpabilité envahissent le moi :

Le départ de Richard Rivière l'avait remplie de honte parce qu'elle avait compris qu'elle avait échoué dans sa volonté d'offrir tout l'amour et toute la mansuétude dont un être pût avoir besoin, et même par-delà le besoin ${ }^{32}$.

La honte est explicitement liée ici la reconnaissance de ses fautes (matérialisée par la locution conjonctive de causalité).

Le temps ne demeure pas son meilleur allié dans l'oubli de cet événement traumatique $^{33}$. Plus le temps passe, plus l'absence se fait déchirure. La protagoniste, «au bord des larmes, rêveuse et comme morte ${ }^{34}$ ne sachant plus que faire de son désespoir, va se mettre au service de son autodestruction. La désunion est envisagée comme un mouvement actif, l'envers de l'amour naissant. Elle se laisse emporter dans une relation toxique matérialisée par la figure chthonienne de Freddy Moliger qui déclenche le commencement de la descente aux enfers de Clarisse. La chute est accélérée et le mal qui va atteindre Clarisse redevenue Malinka, est annoncé par la figure de Freddy qui "s'était approché d'elle avec sa figure d'infortune, son corps branlant, abîmé, d'une maladive

\footnotetext{
${ }^{30}$ Ibidem, p. 64.

${ }^{31}$ Juan-David Nasio, La douleur d'aimer, p. 225.

${ }^{32}$ Ibidem, p. 99.

${ }^{33} \mathrm{Si}$ l'abandon fait si mal à Clarisse, c'est parce qu'elle a déjà vécu une expérience traumatique auparavant. Sa rupture amoureuse ne constitue pas en effet sa première expérience de l'abandon. Elle affirme elle-même : « Mon père ne m'avait pas reconnue » (Marie NDiaye, Ladivine, p. 66).

${ }^{34}$ Ibidem, p. 95.
} 
maigreur $»^{35}$. Ce dernier (on saura à la fin qu'il est l'assassin de Clarisse), dont le portrait s'affuble des qualificatifs les plus abjects, est décrit comme repoussant voire répugnant :

Elle (Malinka) regardait ses yeux délavés, aux coins tombants, la peau grêlée et marbrée de son visage, ses cheveux jaunes et rêches qui lui faisaient penser à un bout de gazon brûlé par le désherbant, elle le regardait et songeait qu'il était ardu d'aimer et de désirer toucher une figure aussi abîmée... ${ }^{36}$.

À partir de la description du portrait physique du personnage masculin, la vue de ce corps qui a atteint un état de délabrement extrême, devrait susciter chez le regardant un sentiment de dégoût et de répulsion. Contrairement à ce qui est attendu, Clarisse se contraint à approcher et à caresser cette figure repoussante :

Elle n'en éprouverait pas le plaisir sensuel qu'elle avait toujours eu à caresser le beau visage sain de Richard Rivière mais elle saurait aimer le faire pourtant, même sans en éprouver de plaisis ${ }^{37}$.

"Rien de ce qui concernait Freddy Moliger n'était agréable ${ }^{38}$ et pourtant Malinka persiste à poursuivre sa relation avec lui. Elle lui propose même de s'installer chez elle, s'exposant ainsi imprudemment au danger.

La volonté d'établir une étroite proximité avec Freddy et de réduire au maximum le degré d'exposition à son futur bourreau, serait une compulsion à l'autopunition (forme de réparation du chagrin). Ne se sentant pas digne de Richard et suite à la perte narcissique, Malinka, avec cette ultime action qu'elle effectue avec une certaine quiétude, démontre, suite à son échec amoureux, qu'elle ne mérite plus considération ni estime :

Elle constatait sa propre négligence et en éprouvait parfois une dure satisfaction, car son corps lui semblait être un vieux chien qu'on ne châtierait jamais assez pour avoir, par exemple, dévoré un petit enfant. Elle se mit à vivre dans l'attente de mourir, épuisée de douleur et d'écœurement de tout... ${ }^{39}$.

Le désir de mort se révèle être d'une intensité extraordinaire et est la conséquence de l'accomplissement d'une faute irrémissible, imagée ici grâce à l'allégorie du chien tueur d'enfant. Il atteste également que le sentiment de mépris de soi et de culpabilité atteigne leur apogée et que rien ne pourra le dissoudre. C'est ce sentiment de sous-estimation de soi qui mènera le personnage à sa perte. Le déni de l'échec amoureux se transforme au fil des pages du roman en déni de soi qui atteint son apogée avec la mort programmée de Clarisse/ Malinka, une mort

\footnotetext{
${ }^{35}$ Ibidem, p. 108.

${ }^{36}$ Ibidem, p. 113.

${ }^{37}$ Ibidem, p. 114.

${ }^{38}$ Ibidem.

${ }^{39}$ Ibidem, p. 99.
} 
qui pourrait être envisagée comme un suicide par procuration. Dans cette perspective, Éros et Thanatos se sont inévitablement alliés. Le mouvement de mépris de soi incite Clarisse à rompre ses attaches avec son propre corps et l'emporte vers une catastrophe salutaire, une inéluctable finitude.

\section{Conclusion}

La violence de la séparation amoureuse, occasionnant simultanément des troubles psychologiques et physiologiques insoutenables chez le personnage délaissé et la description de symptômes alimentant la progression du récit, pourrait s'apparenter à une plaie vive, inguérissable qui abandonne le sujet malheureux dans un état d'angoisse profonde et destructrice ${ }^{40}$. Ainsi, le texte ndiayïen, dont la trame narrative épouse les trajectoires émotionnelles de son personnage, offre l'exemple de la dépendance et de l'attachement affectifs, qui ne se résout que dans le suicide de son personnage. Rupture amoureuse pourrait donc être synonyme de coupure irréversible non seulement avec l'autre qui part, mais également avec son propre corps.

\section{BIBLIOGRAPHIE}

BARTHES, Roland, «Fragments d'un discours amoureux », in CEuvres complètes, V, Paris, Seuil, 1977.

FREUD, Sigmund, La naissance de la psychanalyse, Paris, PUF, 1991.

FREUD, Sigmund, «Au-delà du principe de plaisir », in Essais de psychanalyse, Paris, Payot, 1971.

KRISTEVA, Julia, Histoires d'amour, Paris, Denoël, 1983.

NASIO, Juan-David, La douleur d'aimer, Paris, Payot, 2005.

NASIO, Juan-David, Le livre de LA DOULEUR et de L'AMOUR, Paris, Payot \& Rivages, 1996.

NDIAYE, Marie, Papa doit manger, Paris, Éditions de Minuit, 2003.

NDIAYE, Marie, Trois femmes puissantes, Paris, Gallimard, 2009.

NDIAYE, Marie, Ladivine, Paris, Gallimard, 2013.

SCHURMANS, Daniel, L'homme qui souffre, Paris, PUF, 2010, p. 28.

\section{LOVE SUFFERING AT MARIE NDIAYE (Abstract)}

The physical as well as the psychological suffering is an omnipresent theme in the work of Marie NDiaye. The writer insists notably on increasing the physical discomfort of his characters in close

\footnotetext{
${ }^{40}$ Roland Barthes (Fragments d'un discours amoureux, p. 42) précise à ce sujet que «l'amoureux qui n'oublie pas quelques fois, meurt par excès, fatigue et tension de mémoire ».
} 
connection with their love disappointment. The pain is lived intensely by the characters that are described through their bodies, surface of capture of the surrounding world and emotional abyss. It will therefore be in the context of this article to detect affective expressions and to study the mechanisms of emotional language. We will also see how the affect shapes the ndiayien character's journey and how it models his narrative environment.

Keywords: Marie NDiaye, pain, love, body, psyche.

\section{SUFERINȚA DIN DRAGOSTE LA MARIE NDIAYE (Rezumat)}

Suferinţa fizică şi emoţională constituie o temă omniprezentă în opera Mariei Ndiaye. Scriitoarea insistă mult pe amplificarea disconfortului fizic al eroilor, în strânsă legătură cu deziluziile lor amoroase. Durerea este trăită intens de personajele descrise prin intermediul corpurilor lor, ca suprafețe de contact cu lumea înconjurătoare şi ca adevărate abisuri emoţionale. În acest context, articolul de faţă se va axa pe detectarea expresiilor afective, pentru a studia mecanismele limbajului emoţional. Vom urmări, de asemenea, felul în care sentimentele determină parcursul personajelor ndiayene, precum şi contextul narativ.

Cuvinte-cheie: Marie Ndiaye, durere, dragoste, trup, suflet. 